\title{
Overview of dermatoses among detainees in the Ouagadougou Correctional Facility (Burkina Faso)
}

\section{Marcellin Bonkoungou', Fagnima Traoré2, Amina Nomtondo Ouédraogo ${ }^{3}$, Muriel Sidnoma Ouédraogo ${ }^{3}$, Gilbert Patrice Tapsoba ${ }^{3}$, Yacinthe Kafando ${ }^{4}$, Séraphine Zeba ${ }^{5}$, Alban Bassolé, Mariam Ludwine Sanou ${ }^{7}$, Salamata Bara ${ }^{8}$, Angèle Ouédraogo ${ }^{3}$, Yaya Ouédraogo ${ }^{9}$, Nina Korsaga/Somé ${ }^{5}$, Jean-Baptiste Andonaba ${ }^{10}$, Fatou Barro/Traoré6 ${ }^{\text {, Antoine Pascal Niamba }}{ }^{3}$, Adama Traoré ${ }^{3}$}

${ }^{1}$ Centre Hospitalier Universitaire Bogodogo, Burkina Faso, ${ }^{2}$ Centre Hospitalier Universitaire Ouahigouya, Burkina Faso, ${ }^{3}$ Centre Hospitalier Universitaire Yalgadogo, Service de Dermatologie, Burkina Faso, ${ }^{4}$ Centre Hospitalier de Dédougou, Burkina Faso, ${ }^{5}$ Hôpital de District de Boulmiougou, Ouagadoudou, Burkina Faso, ${ }^{6}$ Centre Hospitalier Universitaire de Tingandogo, Ouagadougou, Burkina Faso, ${ }^{7}$ Centre Hospitalier Régional de Kaya, Burkina Faso, ${ }^{8}$ Centre Médical avec Antenne Chirurgical de Ziniairé, Burkina Faso, ${ }^{9}$ Centre Hospitalier Régional de Fada, Burkina Faso, ${ }^{10}$ Centre Hospitalier Sourou Sanou, Bobo, Burkina

Corresponding author: Dr. Marcellin Bonkoungou, E-mail: bonkoungou_marcelin@yahoo.fr

\begin{abstract}
Background: Very few data are available on the scope of skin and mucosal diseases in Burkina Faso prison environment. The purspose of this study is to determine nosological types, scope and determining factors of these diseases in the biggest detention center of the country. Patients and methodology: On 16 - 20 July, 2018, we conducted a descriptive and analytical cross-cutting study, involving all detainees suffering from one or several dermatoses, and who gave an informed consent. Information collected included types of dermatoses and socio-demographic data. Risk factors surveyed covered hygiene and sanitation, focusing on frequency of showers, use of soap during showers, change of clothes and sanitation of cells. Data were analysed using Epi info 3.5.4 software. For statistics, we used Peasrson Chi-Square Test with 5\% threshold significance. Findings: In total, 1,510 out of 1,916 detainees (78.81\%) were examined. More than 1 detainee in 2, had one or several dermatoses with 839 cases, that is an occurrence of $55.56 \%$. The average age was 31.95 years $\pm 9,35$. Infectious dermatoses were most represented with 870 cases $(67.5 \%)$ and included intertrigos among 357 detainees (27.69\%), scabies among 166 detainees (12.87\%) and ringworms among 143 detainees (11.09\%). Noninfectious dermatoses represented $32.5 \%$ including 114 cases of acne $(8.84 \%)$, and 44 cases of eczema (3.41\%). Almost all detainees $(94.8 \%$ ) presenting a dermatosis, had regular showers. $87 \%$ were regularly using soap during showers and $27.3 \%$ were irregularly changing clothes after showers. Regarding cells, $80 \%$ were irregularly sanitized and we observed carceral overcrowding (297.5\%). None of the patients had a dermatological consultation after their incarceration. Hygiene conditions were significantly associated with occurrence of infectious dermatoses. Conclusion: Dermatoses are frequent in prison environment in Burkina Faso. Infectious ones are predominent. Sanitation of cells and improvement in detention conditions are crucial and should be priorities for the penitentiary authorities of the country. Besides, detainees should receive dermatological consultations on a regular basis.
\end{abstract}

Key words: Infectious dermatoses; Prison; Hygiene; Burkina Faso

\footnotetext{
How to cite this article: Bonkoungou M, Traoré F, Ouédraogo AN, Ouédraogo MS, Tapsoba PG, Kafando Y, Zeba S, Bassolé A, Sanou ML, Bara S, Ouédraogo A, Ouédraogo Y, Korsaga/Somé N, Andonaba J-B, Barro/Traoré F, Niamba AP, Traoré A. Overview of dermatoses among detainees in the Ouagadougou Correctional Facility (Burkina Faso). Our Dermatol Online. 2020;11(e):e93.1-e93.7.

Submission: 20.03.2020; Acceptance: 07.05.2020

DOI: 10.7241 /ourd.2020e.93
} 


\title{
Panorama des dermatoses chez les détenus de la maison d'arrêt et de correction de Ouagadougou (Burkina Faso)
}

\author{
Marcellin Bonkoungou', Fagnima Traoré2, Amina Nomtondo Ouédraogo ${ }^{3}$, \\ Muriel Sidnoma Ouédraogo ${ }^{3}$, Gilbert Patrice Tapsoba ${ }^{3}$, Yacinthe Kafando, \\ Séraphine Zeba ${ }^{5}$, Alban Bassolé 6 , Mariam Ludwine Sanou ${ }^{7}$, Salamata Bara ${ }^{8}$, \\ Angèle Ouédraogo ${ }^{3}$, Yaya Ouédraogo ${ }^{9}$, Nina Korsaga/Somé ${ }^{5}$, Jean-Baptiste Andonaba ${ }^{10}$, \\ Fatou Barro/Traoré ${ }^{\text {, Antoine Pascal Niamba }{ }^{3} \text {, Adama Traoré }}{ }^{3}$
}

${ }^{1}$ Centre Hospitalier Universitaire Bogodogo, Burkina Faso, ${ }^{2}$ Centre Hospitalier Universitaire Ouahigouya, Burkina Faso, ${ }^{3}$ Centre Hospitalier Universitaire Yalgadogo, Service de Dermatologie, Burkina Faso, ${ }^{4}$ Centre Hospitalier de Dédougou, Burkina Faso, ${ }^{5}$ Hôpital de District de Boulmiougou, Ouagadoudou, Burkina Faso, ${ }^{6}$ Centre Hospitalier Universitaire de Tingandogo, Ouagadougou, Burkina Faso, ${ }^{7}$ Centre Hospitalier Régional de Kaya, Burkina Faso, ${ }^{8}$ Centre Médical avec Antenne Chirurgical de Ziniairé, Burkina Faso, ${ }^{9}$ Centre Hospitalier Régional de Fada, Burkina Faso, ${ }^{10}$ Centre Hospitalier Universitaire Sourou Sanou, Bobo, Burkina

Corresponding author: Dr. Marcellin Bonkoungou, E-mail: bonkoungou_marcelin@yahoo.fr

\section{RÉSUMÉ}

Introduction: Il existe peu de données concernant l'ampleur des maladies de la peau et des muqueuses dans le milieu carcéral burkinabè. Lobjectif de cette étude était de déterminer les types nosologiques, l'ampleur et les facteurs déterminants de ces dermatoses dans le plus grand centre de détention du pays. Patients et méthode: Nous avons conduit une étude transversale descriptive et analytique du 16 au 20 juillet 2018 à la Maison d'Arrêt et de Correction de Ouagadougou (MACO). Ont été inclus tous les détenus présentant ou non une dermatose et consentants. Les informations collectées etaient les types de dermatose, les données socio-démographiques et les facteurs de risques recherchés étaient relatifs à hygiène et l'assainissement notamment la fréquence des douches, l'utilisation de savon pour la douche, le changement de vêtements et l'assainissement des cellules. Les données ont été analysées par le logiciel Epi info 3.5.4, et pour les statistiques, nous avons utilisé le test de Chi2 de Pearson avec un seuil de significativité de 5\%. Résultats: Au total,1510 détenus sur 1916 ont été examinés soit 78,81\% de l'effectif. Plus d'un détenu sur 2 souffrait d'une ou plusieurs dermatoses avec 839 cas soit une fréquence de 55,56\%. Lâge moyen était 31,95 ans $\pm 9,35$. Les dermatoses infectieuses étaient les plus représentées, avec 870 cas (67,5\%), et se composaient des intertrigos chez 357 détenus (27,69\%), de la gale chez 166 détenus (12,87\%) et des dermatophyties chez 143 détenus (11,09\%). Les dermatoses non infectieuses représentaient 32,5\% (419) dont 114 cas d'acné (8,84\%), et 44 cas d'eczéma (3,4l\%), La quasi-totalité des détenus $(94,8 \%)$ présentant une dermatose se lavaient régulièrement, $87 \%$ utilisaient régulièrement le savon pendant la toilette, et $27,3 \%$ changeaient irrégulièrement de vêtements après la toilette. Quant aux conditions de détention, $80 \%$ des cellules d'emprisonnement étaient irrégulièrement assainies et on notait une surpopulation carcérale (297,5\%). Aucun des détenus n'avait bénéficié d'une consultation dermatologique après l'incarcération. Les conditions d'hygiène étaient significativement associées à la survenue d'une dermatose infectieuse. Conclusion: Les dermatoses sont fréquentes en milieu carcéral au Burkina Faso, celles infectieuses prédominaient. Lassainissement des cellules et amélioration des condtitions de détention sont essentielles et devraient être des priorités des autorités pénitentiaires du pays. Par ailleurs, ces détenus devraient bénéficier d'une consultation dermatologique régulièrement.

Mots clés : Dermatoses infectieuses; Prison; Hygiène; Burkina Faso

\footnotetext{
How to cite this article: Bonkoungou M, Traoré F, Ouédraogo AN, Ouédraogo MS, Tapsoba PG, Kafando Y, Zeba S, Bassolé A, Sanou ML, Bara S, Ouédraogo A, Ouédraogo Y, Korsaga/Somé N, Andonaba J-B, Barro/Traoré F, Niamba AP, Traoré A. Overview of dermatoses among detainees in the Ouagadougou Correctional Facility (Burkina Faso). Our Dermatol Online. 2020;11(e):e93.1-e93.7.

Submission: 20.03.2020; Acceptance: 07.05.2020

DOI: $10.7241 /$ ourd.2020e.93
} 


\section{INTRODUCTION}

La surpopulation, la promiscuité et le défaut d'hygiène dans les prisons constituent des conditions favorables pour le développement de diverses pathologies notamment celles transmissibles y compris les affections dermatologiques. Parmi ces maladies infectieuses, l'infection à VIH et la tuberculose retiennent l'attention du fait de leur gravité. Par contre, les affections de la peau considérées à tort comme bénignes sont souvent négligées mais cependant elles peuvent être responsables d'une morbidité importante. Plusieurs études menées dans des prisons concernant ces affections retrouvaient des fréquences variées d selon le niveau de développement du pays [1-4]. On notait 7,7\% dans les prisons belges [5], 27\% dans les prisons en Suisse [6], 27,3\% en Guinée Conakry [3], 49,2\% au Nigeria [7], 56,2\% au Cameroun [4].

Au Burkina Faso, où les maladies de la peau sont fréquentes et constituent le quatrième motif de consultation dans les structures de soins [8], aucune étude n'a évalué de façon exhaustive leur prévalence en milieu carcéral en dehors des atteintes mycosiques [9]. C'est dans cette optique que nous avons mené cette étude pour en déterminer les types et la fréquence des dermatoses ainsi que leurs déterminants à la Maison dArrêt et de Correction de Ouagadougou (MACO), la plus grande structure de détention du Burkina Faso. Nos résultats pourraient permettre d'élaborer des stratégies pour améliorer la santé des détenus dans les prisons au Burkina Faso.

\section{PATIENTS ET MÉTHODE}

\section{Type et durée d'étude}

Il s'est agi d'une étude transversale réalisée du 16 au 20 juillet 2018. Ont été inclus tous les détenus de la MACO consentants qui se sont présentés lors de la consultation.

\section{Site d'étude}

La MACO a été construite en 1962. Elle a connu une extension de ses capacités d'accueil en 1994 et en 2010 avec la construction d'autres " quartiers" pour pallier au surpeuplement, à la cohabitation entre adultes et enfants, a supprimer. et de sexes opposés. Elle comprend depuis lors 5 quartiers: 2 quartiers pour les hommes adultes, un quartier pour les détenus du monde politique et économique, un quartier pour les femmes adultes et pour les mineurs. Sa capacité d'accueil théorique est de 570 détenus en 2010

\section{Déroulement de l'étude}

Sur la période d'étude, nous avons mobilisé 14 dermatologues bénévoles qui ont assuré les consultations des détenus. Le diagnostic reposait exclusivement sur des arguments anamnestiques et cliniques. Les détenus chez lesquels une dermatose était diagnostiquée recevait des médicaments mis à la disposition par une Organisation Non Gouvernementale (ONG), Expertise France Burkina. Les détenus pour lesquels des explorations complémentaires étaient nécessaires ont bénéficié d'un accompagnement financier en collaboration avec l'administration pénitentiaire et la dite ONG pour la prise en charge médicale. Un suivi médical était organisé avec les agents de santé de l'établissement pénitentiaire pour ceux dont le tableau clinique nécessitait un continuum de soins au-delà de la période de la dite activité.

Les variables collectées étaient celles sociodémographiques (l'âge, le sexe, l'état civil) les conditions de détentions (la durée de l'incarcération, le nombre de détenus par cellule)l'hygiène (la fréquence des douches quotidiennes, l'utilisation de savon pour la douche, le changement de vêtements), les types des dermatoses.

Les données ont été analysées par le logiciel Epi info 3.5.4, et pour les statistiques, nous avons utilisé le test de Chi2 de Pearson avec un seuil de significativité de 5\%

\section{Considération éthique}

Une autorisation a été fournie par les autorités pénitentiaires et judiciaires du Burkina Faso pour réaliser l'étude. Le consentement éclairé des détenus était obtenu avant leur inclusion dans l'étude.

\section{RÉSULTATS}

Sur un total de 1916 détenus, 1510 (78,81\%.) étaient vus par les dermatologues. Parmi eux, 839 présentaient une ou plusieurs dermatoses soit une fréquence de $55,56 \%$. Il y avait 826 hommes et 13 femmes. Lâge moyen était de 31,95 ans $\pm 9,35$ avec des extrêmes de 14 et 87 ans. Tous les détenus n'avaient jamais bénéficié de consultation dermatologique pendant l'incarcération. Quant aux antécédents de dermatoses, $4 \%$ déclaraient en avoir eu avant l'incarcération. Les caractéristiques 
socio-economiques des patients sont résumées dans le Tableau 1.

Sur le plan de l'hygiène et assainissement, 94,8 $\%$ des détenus présentant une dermatose se douchaient régulièrement, $87 \%$ des détenus utilisaient régulièrement le savon pendant la douche, et $27,3 \%$ changeaient irrégulièrement les vêtements après la douche. La distribution des dermatoses en fonction des facteurs de risque est résumée dans le Tableau 2.

Quant aux cellules 80 \% étaient irrégulièrement assainies et on notait une surpopulation carcérale $(297,5 \%)$.

Les dermatoses infectieuses étaient les plus représentées, avec 870 cas $(67,5 \%)$, et se composaient des intertrigos chez 357 détenus $(27,69 \%)$, de la gale chez 166 détenus $(12,87 \%)$ et des dermatophyties chez 143 détenus (11,09\%). Les dermatoses non infectieuses représentaient 32,5\% (419) dont 114 cas d'acné $(8,84 \%)$, et 44 cas d'eczéma $(3,41 \%)$. Le Tableau 3 et 4 résument la distribution des dermatoses rencontrées. Les Figures la, 1b, 2a et $2 \mathrm{~b}$ montrent respectivement une gale et une dermatophytie profuse.

Tableau 1: Caractéristique socio démographique [Socio demographic characteristic]

\begin{tabular}{lcc}
\hline Variable & Nombre & Proportion \\
\hline AGE & 50 & \\
$<20$ & 359 & 6 \\
$20-29$ & 312 & 42,1 \\
$30-39$ & 88 & 37,2 \\
$40-49$ & 26 & 10,5 \\
$50-59$ & 10 & 3,10 \\
Superieur 60 & & 1,2 \\
SEXE & 826 & \\
$\quad$ Masculin & 13 & 98,5 \\
Feminin & & 1,5 \\
Durée incarcération & 106 & \\
$<1$ an & 622 & 12,7 \\
1-5 ans & 111 & 74,1 \\
Superieur à 5 ans & & 13,2 \\
\hline
\end{tabular}

\section{DISCUSSION}

Cette étude sur les dermatoses en milieu carcéral est une première au Burkina Faso. Elle a révélé une prévalence globale de 55,56\%. Nos résultats sont similaires à ceux rapportés par d'autres auteurs, à savoir une prévalence élevée des dermatoses dans la population carcérale.

Les jeunes adultes sont ceux qui prédominent dans les prisons, certainement en raison du chômage et qui est source de pauvreté dans cette tranche d'âge au Burkina Faso. Ils sont ainsi plus enclins à la délinquance et aux comportements répréhensibles. En outre, le rapport de masculinité que nous avons obtenu $(826$ hommes et 13 femmes) est comparable aux observations de la prison principale de Mfou et de la prison de Ngaoundéré (région d'Adamaoua au Cameroun), dans la région occidentale du Cameroun [4,10,], et à Conakry [3] .

La prévalence des dermatoses correspond à celles rapportées par Kouotou et al. dans la région du centre du Cameroun 57,1\% [10], dans la région occidentale du Cameroun [4] 56.2\%, par Oninla OA et al au Nigeria 49,2\% [7] , 55,7\% obtenus par Roodsari et al., à la prison de Ghezel Hesar, Iran [11] et les 63,63 par M Kuruvila et al. en Inde [12]. Nos résultats sont supérieurs à ceux de Parajuli et al., [13] au Népal, avec une prévalence de dermatose de $27.2 \%$ de même que celui de Tounkara et al., [3] 27.3\% en Guinée.

Cette prévalence élevée de pathologies dermatologiques dans les pays sous développés peut êtree due à la surpopulation carcérale et aux conditions climatiques tropicales chaudes (températures au dessus de $30^{\circ}$ ) favorisant le développement de bactéries et de champignons.

Contrairement à la tendance observée dans la population générale où les dermatoses immunoallergiques dominent les affections cutanées [14], la présente étude note plutôt une prédominance des

Tableau 2: Distribution des dermatoses en fonction des facteurs de risque [Distribution of dermatoses according to risk factors]

\begin{tabular}{|c|c|c|c|c|}
\hline Factores & Dermatoses oui & Dermtose non & Chi-square & $\mathbf{P}$ \\
\hline \multicolumn{5}{|c|}{ Utilisation de savon pour la toilette } \\
\hline Regulier & 730 & 537 & 14.7679 & $<0.0001$ \\
\hline Irrégulier & 109 & 37 & & \\
\hline \multicolumn{5}{|c|}{ Fréquence de la toilette } \\
\hline Régulier & 796 & 636 & 0.0063 & 0.936761 \\
\hline irrégulier & 43 & 35 & & \\
\hline \multicolumn{5}{|c|}{ Changement d'habit après la douche } \\
\hline Oui & 610 & 466 & 1.9311 & 0.164641 \\
\hline Non & 229 & 205 & & \\
\hline
\end{tabular}




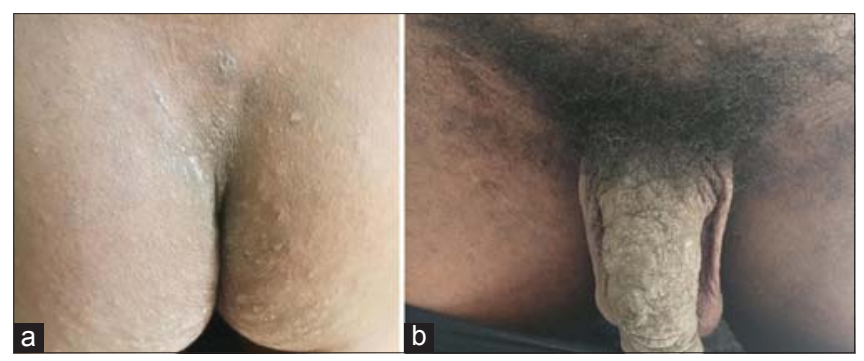

Figures 1a et 1b: Gale profuse chez un détenu [Diffuse scabies in an detainee].

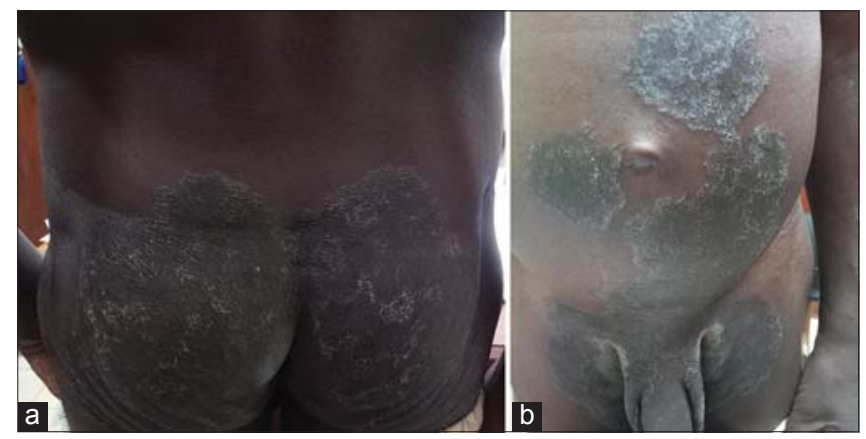

Figures 2a et 2b: Dermatophytie profuse [Diffuse dermatophytosis].

dermatoses infectieuses. Des tendances similaires ont déjà été signalées dans des études antérieures menées dans la région du centre du Cameroun 76,4\% [10], en Guinée 77,2\%, au Togo $51 \%$, au Nigéria 53,6\%, et en Inde $63.3 \%$.

Les dermatoses infectieuses les plus rencontrées étaient les intertrigos $27,69 \%$, la gale $12,87 \%$, les dermatophyties $11,09 \%$ et le ptyriasis versicolor 7,91\%. Dans notre étude, comme dans celles de plusieurs auteurs, les atteintes mycosiques ont également une prévalence élevée en milieu carcéral $[3,9,15,16]$. La surpopulation carcérale, la promiscuité et les mauvaises conditions d'hygiène, sont autant de facteurs qui favorisent cette forte représentativité. Les salles de détention sont caractérisées par un environnement chaud, humide, peu aéré notamment dans les deux bâtiments des hommes adultes. Toute chose qui favorise la transpiration excessive et son corollaire d'intertrigo mycosique, de pityriasis versicolore, de dermatophytie.

La prévalence élevée de la gale a été retrouvée par plusieurs auteurs dans les prisons africaines $[3,4,10]$. Cette prévalence de la scabiose dans nos prisons suggère un contact fréquent et prolongé entre détenus, sachant que la transmission de la gale se fait principalement par contact interhumain. En effet, il a été prouvé que la surpopulation, la promiscuité, une hygiène médiocre, une qualité de vie précaire et l'absence totale de soins de santé constituent des facteurs de risque importants pour
Tableau 3: Panorama des dermatoses [Overview of dermatoses]

\begin{tabular}{|c|c|c|c|}
\hline & $\begin{array}{c}\text { Total } \\
\mathrm{N}=1289\end{array}$ & $\begin{array}{c}\text { Proportion } \\
\text { par groupe de } \\
\text { dermatose } \%\end{array}$ & $\begin{array}{c}\text { Proportion } \\
\%\end{array}$ \\
\hline \multicolumn{4}{|l|}{ Dermatoses infectieuses } \\
\hline Gale & 166 & 19 & 12,878 \\
\hline Intertrigo & 357 & 41 & 27,696 \\
\hline Pityriasis versicolor & 102 & 12 & 7,913 \\
\hline Furoncle & 49 & 6 & 3,801 \\
\hline Folliculite & 41 & 5 & 3,181 \\
\hline Varicelle & 12 & 1 & 0,931 \\
\hline Dermatophytie & 143 & 16 & 11,094 \\
\hline Sous total & 870 & & \\
\hline \multicolumn{4}{|c|}{ Dermatoses immuno allergique et inflammatoires } \\
\hline Eczéma & 44 & 20 & 3,413 \\
\hline Acné & 114 & 51 & 8,844 \\
\hline Dermite séborrhéique & 12 & 5 & 0,931 \\
\hline Dermite d'irritation & 6 & 3 & 0,465 \\
\hline Prurigo & 41 & 18 & 3,181 \\
\hline Névrodermite & 6 & 3 & 0,465 \\
\hline Sous Total & 223 & & \\
\hline \multicolumn{4}{|l|}{ Dermatose tumorale } \\
\hline Chéloïde & 20 & 61 & 1,552 \\
\hline Kyste & 6 & 18 & 0,465 \\
\hline Molluscum pendulum & 1 & 3 & 0,078 \\
\hline $\begin{array}{l}\text { Dermatosis papulosa } \\
\text { nigricans }\end{array}$ & 6 & 18 & 0,465 \\
\hline Sous Total & 33 & & \\
\hline \multicolumn{4}{|l|}{ Autres dermatoses } \\
\hline Prurit & 138 & 87 & 10,706 \\
\hline Miliaire sudorale & 15 & 9 & 1,164 \\
\hline Ulcère de jambe & 2 & 1 & 0,155 \\
\hline Lichenification & 2 & 1 & 0,155 \\
\hline Xérose & 2 & 1 & 0,155 \\
\hline Sous Total & 159 & & \\
\hline \multicolumn{4}{|l|}{ IST } \\
\hline Ecoulement urétral & 3 & 75 & 0,233 \\
\hline Végétation vénérienne & 1 & 25 & 0,078 \\
\hline Ulcération génital & 0 & 0 & 0,000 \\
\hline Douleur pelvienne & 0 & 0 & 0,000 \\
\hline Sous Total & 4 & & \\
\hline Total & 1289 & 100 & 100 \\
\hline
\end{tabular}

la survenue et la propagation de la gale humaine. Dans l'étude de B. Khatibi et al., en France, le faible taux de gale était dû au traitement anti-scabieux et à l'isolement pendant une semaine des prisonniers pour lesquels ce diagnostic avait été évoqué lors de la consultation médicale systématique qui a suivi leur transfert de leur lieu de détention [17]. Aussi la prévalence élevée des maladies cutanées infectieuses pourrait également être alimentée par la prévalence élevée (3.58\%) de l'infection à VIH dans le milieu [18], étant donné que le virus affaiblit le système immunitaire.

Les dermatoses non infectieuses étaient dominées par l'acné et l'eczéma. La survenue d'acné chez les détenus peut être due au stress accru inhérent à la vie en prison, 
Tableau 4: Panorama des dermatoses en fonction des tranches d'âges [Overview of dermatoses according to age groups]

\begin{tabular}{|c|c|c|c|c|c|c|c|}
\hline & Moins de 20 ans & $20-29$ ans & 30-39ans & 40-49ans & $50-59$ ans & Plus de 59ans & $N=1289$ \\
\hline \multicolumn{8}{|l|}{ Dermatoses infectieuses } \\
\hline Gale & 13 & 61 & 65 & 17 & 6 & 4 & 166 \\
\hline Intertrigo & 14 & 145 & 140 & 44 & 12 & 2 & 357 \\
\hline Pityriasis versicolor & 7 & 46 & 40 & 8 & 0 & 1 & 102 \\
\hline Furoncle & 4 & 15 & 25 & 4 & 1 & 0 & 49 \\
\hline Folliculite & 3 & 14 & 18 & 4 & 1 & 1 & 41 \\
\hline Varicelle & 1 & 5 & 6 & 0 & 0 & 0 & 12 \\
\hline Dermatophytie & 15 & 60 & 54 & 9 & 4 & 1 & 143 \\
\hline \multicolumn{8}{|c|}{ Dermatoses immuno allergique et inflammatoires } \\
\hline Eczéma & 3 & 12 & 18 & 8 & 2 & 1 & 44 \\
\hline Acné & 9 & 58 & 38 & 8 & 1 & 0 & 114 \\
\hline Dermite séborrhéique & 1 & 4 & 5 & 1 & 0 & 1 & 12 \\
\hline Dermite d'irritation & 1 & 0 & 4 & 0 & 1 & 0 & 6 \\
\hline Prurigo & 2 & 17 & 19 & 2 & 1 & 0 & 41 \\
\hline Névrodermite & 1 & 1 & 4 & 0 & 0 & 0 & 6 \\
\hline \multicolumn{8}{|l|}{ Dermatose tumorale } \\
\hline Chéloïde & 1 & 6 & 11 & 0 & 1 & 1 & 20 \\
\hline Kyste & 1 & 0 & 5 & 0 & 0 & 0 & 6 \\
\hline Molluscum pendulum & 0 & 0 & 1 & 0 & 0 & 0 & 1 \\
\hline Dermatosis papulosa nigricans & 1 & 0 & 5 & 0 & 0 & 0 & 6 \\
\hline \multicolumn{8}{|l|}{ Autres dermatoses } \\
\hline Prurit & 7 & 54 & 56 & 10 & 5 & 6 & 138 \\
\hline Miliaire sudorale & 2 & 6 & 7 & 0 & 0 & 0 & 15 \\
\hline Ulcère de jambe & 0 & 0 & 1 & 0 & 0 & 1 & 2 \\
\hline Lichenification & 0 & 0 & 2 & 0 & 0 & 0 & 2 \\
\hline Xérose & 0 & 0 & 2 & 0 & 0 & 0 & 2 \\
\hline \multicolumn{8}{|l|}{ IST } \\
\hline Ecoulement urétral & & 1 & 2 & 0 & 0 & 0 & 3 \\
\hline Végétation vénérienne & 0 & 0 & 0 & 0 & 1 & 0 & 1 \\
\hline Ulcération génital & 0 & & 0 & 0 & 0 & 0 & 0 \\
\hline Douleur pelvienne & 0 & 0 & 0 & 0 & 0 & 0 & 0 \\
\hline
\end{tabular}

mais plus encore à la prédominance des jeunes dans le milieu. Ces derniers étant connus comme des facteurs de risque pour le développement de l'acné. Bien que l'eczéma puisse survenir chez toute personne, il a été démontré que des conditions de l'environnement telle que la chaleur favorise la diffusion de l'allergène [19]. Cette dermatose immunoallergique a été retrouvé par plusieurs auteurs $[3,10,19]$.

D'autres dermatoses ont également été notées: chélö̈des $(1,55 \%)$, folliculite $(3,18 \%)$, prurigo $(3,18 \%)$, miliaires sudorales $(1,16 \%)$, prurits $(10,70 \%)$ et dermatite séborrhéique $(0,93 \%)$. Cela reflète de la diversité des affections cutanées dans les prisons africaines, $[2,10,12,16]$.

Les chéloïdes étaient surtout post traumatiques, rendant compte des traumatismes corporels subis en détention. Le prurit sans lésions dermatologiques, les exportations complémentaires n'ont pas été possibles pour en déterminer les étiologies. Néanmoins, l'hyperhidrose en est une cause fréquente.
En ce qui concerne les conditions d'hygiène, seulement $20 \%$ des cellules de la prison étaient assainies quotidiennement, $5,2 \%$ des prisonniers prenaient une douche irrégulière, $13 \%$ se douchaient sans savon et $72,7 \%$ changeaient des vêtements après la douche. Nous avons trouvé une relation statistiquement significative entre les conditions d'hygiène en prison et certains paramètres tel que l'utilisation du savon pendant la douche et la survenue d'une dermatose infectieuse avec $\mathrm{p}<0,05$. Oninla $\mathrm{OA}$ et al., Makoutode $\mathrm{M}$ et al., et Tounkara et al dans leurs études respectives, ont établi l'existence d'un lien statistiquement significatif entre les conditions d'hygiène de l'environnement carcéral y compris la fréquence des douches, l'utilisation de savon, la fréquence des changements de vêtements et la survenue d'une dermatose infectieuse [3,16,20].

Cette étude, qui a concerné le plus grand établissement pénitentiaire du Burkina, ne peut être extrapolé à l'ensemble du pays, mais compte tenu des conditions socio-économiques, le transfert de certains détenus des régions vers la MACO ou vice versa nous émettons 
l'hypothèse que le profil décrit ici serait comparable à celui des autres centres de détention du pays. Une étude plus large incluant les prisons des régions pourrait confirmer ou infirmer notre hypothèse.

\section{CONCLUSION}

Cette étude a montré que les dermatoses surtout infectieuses sont très fréquentes en milieu carcéral et leur profil constitue un important marqueur des conditions carcérales au Burkina. IL n'est pas différent de celui de la majorité des pays à ressources limitées et ce sont des affections courantes et bénignes. Une prévention basée sur les mesures d'hygiène et l'assainissement, une politique carcérale qui désengorge les prisons, des services de santé carcéraux renforcés sont nécessaires pour réduire les pathologies dermatologiques au Burkina Faso. La télé dermatologie entre l'équipe de santé et les dermatologues pourrait faciliter l'accès aux soins dermatologiques des détenus

\section{Remerciements}

Nos remerciements s'adressent à la Société Burkinabè de dermatologie et de Cosmétologie (SOBUDEC), à Expertise France Burkina ainsi qu'à l'administration de la Maison d'Arrêt et de Correction de Ouagadougou.

\section{Statement of Human and Animal Rights}

All procedures followed were in accordance with the ethical standards of the responsible committee on human experimentation (institutional and national) and with the Helsinki Declaration of 1975, as revised in 2008.

\section{Statement of Informed Consent}

Informed consent was obtained from all patients for being included in the study.

\section{RÉFÉRENCES}

1. Roodsari R, Malekzad F, Ardakani ME. Skin diseases in male prisoners. Indian J Dermatol Venereol Leprol. 2007;73:55-6.

2. Akakpo AS, Ekouevi DK, Toure AM, Saka B, Sogan A, d'Almeida S, et al. Skin disease and HIV infec on among inmates in Lome, Togo: a study of 194 prisoners. Med Sante Trop. 2014;24:326-8.

3. Tounkara TM, Soumah MM, Keita M, Sako FB, Traoré FA, Diallo T, et al. Dermatological pathology in an African prison environment: Guinean experience. Dermatol Aspects. 2017;5:1.

4. Kouotou EA, Adegbidi H, Nansseu JRN, Bogne LM, Sieleunou I,
Tatsa JT, et al. Epidemiological and clinical pro les of skin diseases in sub-saharan african prisons: a crosssec onal study from the West Region of Cameroon. Clin Dermatol. 2016;4:46-51.

5. Feron JM, Paulus D, Tonglet R, Lorant V, Pestinaux D. Substantial use of primary health care by prisoners: epidemiological description and possible explanations. J Epidemiol Community Health. 2005;59:651-5.

6. Wolff H, Sebo P, Haller DM, Eytan A, Niveau G, Bertrand D, et al. Health problems among detainees in Switzerland: a study using the ICPC-2 classification. BMC Public Health. 2011;11:245.

7. Oninla OA, Onayemi O, Olasode OA, Oninla SO. Pattern of dermatoses among inmates of Ilesha Prison, Nigeria. Niger Postgrad Med J. 2013;20:174-80.

8. Ministere de la santé du Burkina Faso. Annuaire satistique 2018, mai 2019:213.

9. Zida A, Barro-Traoré F, Dera M, Bazie Z, Niamba P, Guiguemde TR. Epidemiological and etiolological aspects of superficial fungal infections among prison inmates in Ouagadougou, Burkina Faso. J Mycol Med. 2015;25:e73-9.

10. Kouotou EA, Sieleunou I, Defo D, Nansseu N. JR, Atenkeng Apasew H, Moyou Somo R, et al. Skin Diseases Among SubSaharan African Prisoners: The Cameroonian Profile. Int J Clin Dermatol Res. 2014;2:701.

11. Roodsari MR, Malekzad F, Ebrahimzadeh M, Ardakani BAA, Ghoraishian M. Prevalence of scabies and pediculosis in Ghezel Hesar prison, Iran. J Pak Assoc Dermatol. 2006;16:201-4.

12. Kuruvila M, Shaikh MI, Kumar P. Pattern of dermatoses among inmates of district prison Mangalore. Indian J Dermatol Venereol Leprol. 2002;68:16-8.

13. Parajuli N, Jonkman-Veenstra G, Jonkman M. Skin Diseases in a Nepali Prison. J Chitwan Med College. 2014;4:1.

14. Andonaba JB, Diallo B, Sakana L, Niamba P. Aspects épidémiologiques des affections dermatologiques au Centre Hospitalier Universitaire Sourou Sanou de Bobo-Dioulasso. Ann Afr Med. 2010;4:668-77.

15. Bayle P, Cuzin L, Paul C, Blanc A, Grill S, Rouge D, et al. Prisoners and skin diseases in Toulouse, France: epidemiological analysis and evaluation of life impact. J Eur Acad Dermatol Venereol. 2009;23:52-7.

16. Oninla $\mathrm{OA}$, Onayemi $\mathrm{O}$. Skin infections and infestations in prison inmates. Int J Dermatol. 2012;51:178-81.

17. Khatibi B, Bambe A, Chantalat C, Resche-Rigon M, Sanna A, Fac C, et al. Teledermatology in a prison setting: A retrospective study of 500 expert opinions. Ann Dermatol Venereol. 2016;143:418-22.

18. Conseil National de Lutte contre le SIDA et les IST. Enquête bio comportementale du Vih-sida en milieu carcéral auprès des détenus hommes et femmes au Burkina Faso. Rapport final. Novembre 2017:42.

19. Kanish B, Bhatia A. Pattern of cutaneous diseases in inmates of central jail, Ludhiana, Punjab. J Evol Med Dent Sci. 2014;3:3679-81.

20. Makoutode M, Dogmo S, Ouendo E-M, Agossou Th, Bessaoud K. Frequency of pathologies and health risk in the civilian prison of Cotonou in Benin. Méd Afr Noire. 2004;51:105-11.

Copyright by Marcellin Bonkoungou, et al. This is an open access article distributed under the terms of the Creative Commons Attribution License, which permits unrestricted use, distribution, and reproduction in any medium, provided the original author and source are credited.

Source of Support: Nil, Conflict of Interest: None declared. 